\title{
Random-Coil Dimensions and Dipole Moments of Vinyl Chloride Chains
}

\author{
J. E. MARK \\ Department of Chemistry and The Macromolecular Research Center, University of Michigan, Ann Arbor, Michigan 48104
}

(Received 23 July 1971)

\begin{abstract}
A rotational isomeric state model with neighbor interactions is used to calculate mean-square unperturbed dimensions and dipole moments for vinyl chloride chains $\mathrm{CH}_{3}-\left(\mathrm{CHCl}-\mathrm{CH}_{2}-\right)_{x} \mathrm{H}$ having degrees of polymerization $x$ ranging from 1 to 150 and stereochemical structures ranging from perfect syndiotacticity to perfect isotacticity. Conformational energies used in the calculation were those which had been established in an analysis based on the stereochemical equilibration of 2,4-dichloro- $n$-pentane by Flory and Williams. The calculations indicate that the dimensions of poly(vinyl chloride) chains of large $x$ should decrease significantly with increasing isotacticity; this dependence of chain dimensions on stereochemical structure is the opposite of that calculated for vinyl chains having alkyl side groups, such as polypropylene. The dipole moments of poly (vinyl chloride) chains are also predicted to decrease with increasing isotacticity and both the dimensions and dipole moments are expected to decrease with increasing temperature, the largest dependence occurring in the case of perfectly syndiotactic chains. The marked differences in configurational characteristics of poly(vinyl chloride) and polypropylene chains are primarily due to the significant difference in size between $\mathrm{Cl}$ atoms and $\mathrm{CH}_{3}$ groups; the electrostatic interactions present in poly (vinyl chloride) chains are of relatively minor importance. The predicted results for large $x$ are in good agreement with published experimental results on poly (vinyl chloride).
\end{abstract}

\section{INTRODUCTION}

There have been a number of recent theoretical studies of the characteristics of the random-coil configurations of vinyl chains $\mathrm{CH}_{3}-\left[\mathrm{CHR}-\mathrm{CH}_{2}-\right]_{x} \mathrm{H}$ as a function of their stereochemistry, their degree of polymerization $x$, and the nature of their pendant groups R. For example, calculations have been carried out for the mean-square unperturbed dimensions, ${ }^{1-3}$ the fourth moment of the dimensions, ${ }^{3}$ stress-optical coefficients, ${ }^{4-6}$ optical rotatory power, ${ }^{7,8}$ small angle $\mathrm{x}$-ray scattering functions, ${ }^{9}$ depolarization anisotropy in light scattering, ${ }^{10}$ probabilities of conformational sequences and their associated resonances in the proton NMR spectra, ${ }^{11-13}$ rotational isomerization due to macroscopic deformation, ${ }^{14}$ and stereochemical compositions in systems equilibrated by means of isomerization catalysts. ${ }^{15-17}$

To date, however, there have been no reported calculations based on a realistic model, which attempt to characterize the random-coil configurations of vinyl chloride oligomers and polymers $\mathrm{CH}_{3}-[\mathrm{CHCl}-$ $\left.\mathrm{CH}_{2}-\right]_{x} \mathrm{H}$. A recent analysis, ${ }^{17}$ however, based on the equilibrium stereochemical composition of the isomers of 2,4-dichloro- $n$-pentane has provided values of the conformational energies of these chain molecules. It is now therefore possible to calculate a number of their configuration-dependent properties. Of considerable interest is the mean-square dimensions $\left\langle r^{2}\right\rangle_{0}$ unperturbed by long-range interactions and their temperature coefficient. In addition, the presence of significantly large electric dipoles along $\mathrm{C}-\mathrm{Cl}$ bonds permits the study of the mean-square dipole moments $\left\langle\mu^{2}\right\rangle_{0}$ and their temperature dependence, properties not previously calculated in a satisfactory manner for any vinyl chain. Dipole moments assume particular importance in the characterization of chain configurations, since they can be determined experimentally over the entire range of chain length, an advantage not shared by a number of other configuration-dependent properties, such as chain dimensions and stress-optical coefficients. Furthermore, as has already been pointed out, ${ }^{17}$ vinyl chloride oligomers and polymers constitute an unusually interesting class of vinyl chains because of the relatively small size of the pendant substituent, $\mathrm{Cl}$, and the presence of electrostatic contributions to the conformational energies.

In this paper we therefore report results of calculations of $\left\langle r^{2}\right\rangle_{0}$ and $\left\langle\mu^{2}\right\rangle_{0}$ of vinyl chloride chains as a

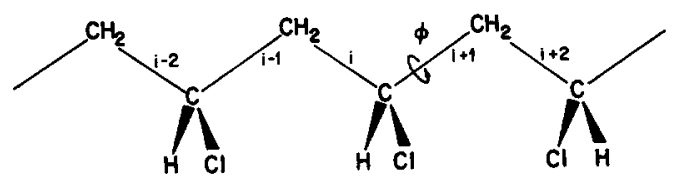

FIG. 1. The planar conformation of a portion of a vinyl chloride chain consisting of an isotactic (dd) dyad followed by a syndiotactic $(d l)$ dyad. Bonds extending toward the reader are represented by lines of increasing thickness, and those extending away, by lines of decreasing thickness. The arrow shows the direction chosen for positive values of the rotational angles $\phi$.

function of their stereochemical structure, degree of polymerization, and temperature. A test of the calculated results in the limit of large $x$ will be provided by a number of previously reported experimental studies on poly (vinyl chloride).

\section{THEORY}

The model adopted for vinyl chloride chains was that previously employed for a number of other chain molecules. ${ }^{18}$ Specifically, each skeletal bond (such as bonds $i-2$ through $i+2$ in Fig. 1) is assumed to occur in any one of three discrete rotational states. In the present case, these states are given the designations 
<smiles>Cc1ccccc1Cl</smiles>

trans, 1
$(\eta)$

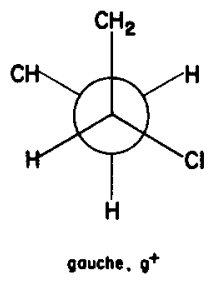

(1)

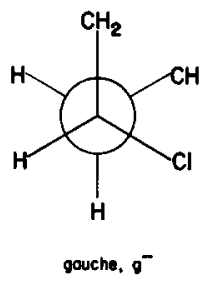

( $\tau$ )
FIG. 2. Newman projections of the three rotational states of $\mathrm{CHCl}-\mathrm{CH}_{2}$ and $\mathrm{CH}_{2}-\mathrm{CHCl}$ skeletal bonds, and the corresponding statistical weight factors.

trans $(t)$, gauche positive $\left(g^{+}\right)$, and gauche negative $\left(g^{-}\right)$and are assumed to occur at rotational angles of $0^{\circ}, 120^{\circ}$, and $-120^{\circ}$, respectively. (Since interactions in this chain are in general not excessively large, ${ }^{17}$ one would not expect displacement of the locations of the rotational states such as occurs in the case of vinyl chains bearing large pendant groups. ${ }^{1}$ )

A number of statistical weight factors are required in order to calculate the configuration-dependent properties of vinyl chloride chains of fixed stereochemical composition. The following scheme is that given by Flory and Williams. ${ }^{16,17}$ We consider first the factors used to characterize "first-order" interactions, i.e., those dependent on only one bond rotational angle. In vinyl chloride chains, these interactions involve $\mathrm{Cl}$ atoms and $\mathrm{CH}_{2}$ and $\mathrm{CH}$ groups, and are described in Fig. 2. The reference state, chosen to be the conformation in which the $\mathrm{CH}$ group is syn to a $\mathrm{CH}_{2}$ group, is assigned a statistical weight factor of unity. A conformation in which this $\mathrm{CH}$ group is syn to the $\mathrm{Cl}$ atom is characterized by the factor $\eta$; the remaining conformation locates the $\mathrm{CH}$ group syn to both the $\mathrm{CH}_{2}$ group and $\mathrm{Cl}$ atom and is associated with the factor $\tau$. Interactions dependent on the rotations of two adjoining skeletal bonds, such as $i-1$ and $i$ in Fig. 1, are said to be of "second order"; they are characterized by the factors $\omega, \omega^{\prime}$, and $\omega^{\prime \prime}$. The first of these, $\omega$, is introduced for conformations engendering interactions between a pair of $\mathrm{CH}_{2}$ groups or $\mathrm{CH}$ groups, separated by four bonds (e.g., $g^{ \pm} g^{\mp}$ states about bonds $i-1$ and $i$, or $g^{ \pm} g^{\mp}$ states about bonds $i$ and $i+1$, respectively). The second factor $\omega^{\prime}$ is associated with second-order interactions between $\mathrm{CH}_{2}$ groups and $\mathrm{Cl}$ atoms (e.g., the $\mathrm{tg}^{+}$state about bonds $i-1$ and $i$ and the $g^{+} t$ state about bonds $i+1$ and $i+2)$. The final factor $\omega^{\prime \prime}$ characterizes secondorder interactions between $\mathrm{Cl}$ atoms (e.g., the $t t$ state about bonds $i-1$ and $i$ and the $t^{-}$state about bonds $i+1$ and $i+2)$. Various combinations of these factors represent statistical weights for all possible conformations about consecutive bond pairs in vinyl chloride chains.

Such statistical weights for pairs of bonds may be succinctly presented in statistical weight matrices $U_{i}$.
Rows in these matrices are associated with rotational states about bond $i-1$ and columns, with states about bond $i$; both are indexed in the order $t, g^{+}$, and $g^{-}$. For a pair of bonds meeting at a $\mathrm{CHCl}$ group, only the atomic configuration of this single group need be specified in the determination of statistical weights. This specification is carried out by the arbitrary designations $d$ and $l$, which are assigned in accordance with the convention shown in Fig. 1. Statistical weight matrices for such bond pairs are designated $\mathbf{U}^{\prime}$ and, for the case of $d$ and $l$ centers, respectively; have the forms ${ }^{1,16}$

$$
\begin{gathered}
\mathbf{U}_{d}=\left[\begin{array}{ccc}
\eta & 1 & \tau \\
\eta & 1 & \tau \omega \\
\eta & \omega & \tau
\end{array}\right], \\
\mathbf{U}_{l}^{\prime}=\left[\begin{array}{lll}
\eta & \tau & 1 \\
\eta & \tau & \omega \\
\eta & \tau \omega & 1
\end{array}\right] .
\end{gathered}
$$

In the case of pairs of bonds separating two $\mathrm{CHCl}$ groups, the atomic configuration of both groups must be specified. The statistical weight matrices for such bond pairs are designated $U^{\prime \prime}$ and, for the four possible dyads, are given by ${ }^{1,16}$

$$
\begin{gathered}
\mathrm{U}_{d d^{\prime \prime}}=\left[\begin{array}{ccc}
\eta \omega^{\prime \prime} & \tau \omega^{\prime} & 1 \\
\eta & \tau \omega^{\prime} & \omega \\
\eta \omega^{\prime} & \tau \omega \omega^{\prime \prime} & \omega^{\prime}
\end{array}\right], \\
\mathbf{U}_{d l} l^{\prime \prime}=\left[\begin{array}{ccc}
\eta & \omega^{\prime} & \tau \omega^{\prime \prime} \\
\eta \omega^{\prime} & 1 & \tau \omega \\
\eta \omega^{\prime \prime} & \omega & \tau \omega^{\prime 2}
\end{array}\right], \\
\mathrm{U}_{l d}{ }^{\prime \prime}=\left[\begin{array}{ccc}
\eta & \tau \omega^{\prime \prime} & \omega^{\prime} \\
\eta \omega^{\prime \prime} & \tau \omega^{\prime 2} & \omega \\
\eta \omega^{\prime} & \tau \omega & 1
\end{array}\right], \\
\mathbf{U}_{l l}^{\prime \prime}=\left[\begin{array}{lll}
\eta \omega^{\prime \prime} & 1 & \tau \omega^{\prime} \\
\eta \omega^{\prime} & \omega^{\prime} & \tau \omega \omega^{\prime \prime} \\
\eta & \omega & \tau \omega^{\prime}
\end{array}\right] .
\end{gathered}
$$

The configuration partition function for the entire chain of $n$ bonds, or $x=\frac{1}{2} n$ repeat units, is then 
simply ${ }^{1,3,18}$

$$
Z=\mathrm{J}^{*}\left(\prod_{i / 2=1}^{n / 2-1} \mathrm{U}_{i}^{\prime} \mathrm{U}_{i+1}{ }^{\prime \prime}\right) \mathrm{J}
$$

where $J^{*}=[100]$ and $\mathrm{J}$ is the transpose of [111]. Calculation of mean-square moments $\left\langle V^{2}\right\rangle$ of any vectorial quantity $\mathbf{v}$ such as the skeletal bond vector 1 or bond dipole vector $m$ requires construction of Cartesian coordinate systems about each bond in the chain backbone. If the $x$ axis is taken along bond $i$, and the positive $y$ axis is taken in the plane determined by bonds $i$ and $i-1$ so as to make an acute angle with bond $i-1$, then the matrix $\mathbf{T}$ which transforms a vector in coordinate system $i+1$ into that of system $i$ is ${ }^{1,18}$

$$
\mathbf{T}_{i}=\left[\begin{array}{ccc}
\cos \theta_{i} & \sin \theta_{i} & 0 \\
\sin \theta_{i} \cos \phi_{i} & -\cos \theta_{i} \cos \phi_{i} & \sin \phi_{i} \\
\sin \theta_{i} \sin \phi_{i} & -\cos \theta_{i} \sin \phi_{i} & -\cos \phi_{i}
\end{array}\right]
$$

where $\theta$ is the bond angle supplement. The rotational angle $\phi$ is defined relative to a value of zero for the trans state, with positive values corresponding to right-handed rotations, as illustrated in Fig. 1. The mean-square moment is then given by ${ }^{1,18}$

$$
\left\langle V^{2}\right\rangle=2 Z^{-1} I^{*} \mathbf{G}_{1}\left(\prod_{i / 2=1}^{n / 2-1} \mathbf{G}_{i}{ }^{\prime} \mathbf{G}_{i+1}{ }^{\prime \prime}\right) \mathbf{G}_{n}{ }^{\prime} \mathbf{I}
$$

where $I^{*}$ is the row vector consisting of a single unity followed by 14 zeros, and $I$ is the column vector consisting of 12 zeros followed by three unities. The generator matrix $\mathbf{G}$ has the form ${ }^{18}$

$$
\mathbf{G}_{i}=\left[\begin{array}{ccc}
\mathbf{U} & \left(\mathbf{U} \otimes \mathbf{v}^{T}\right)\|\mathbf{T}\| & \left(\nu^{2} / 2\right) \mathbf{U} \\
\mathbf{0} & \left(\mathbf{U} \otimes \mathbf{E}_{3}\right)\|\mathbf{T}\| & \mathbf{U} \otimes \mathbf{v} \\
0 & 0 & \mathbf{U}
\end{array}\right]_{i},
$$

where the subscript on the brackets refers to all quantities contained therein; as in the case of the statistical weight matrices, the prime or primes on $\mathbf{G}$ specifies the type of bond pair. The symbol $\otimes$ designates the matrix direct product, ${ }^{18}$ and $\nabla^{T}$ and $v$ are the transpose and magnitude, respectively, of the vector quantity under consideration. The matrices $\mathbf{G}_{1}$ and $\mathbf{G}_{n}{ }^{\prime}$ are atypical since they characterize the first and last bonds, respectively, of the chain; the statistical weight matrix in these two generator matrices is simply the unit matrix $\mathbf{E}_{3}$ of order three. The pseudodiagonal matrices $\left\|\mathbf{T}_{i}\right\|$ are defined by

$$
\left\|\mathbf{T}_{i}\right\|=\left[\begin{array}{ccc}
\mathbf{T}_{i}\left(\phi_{i}\right) & \cdot & \\
& \mathbf{T}_{i}\left(\phi_{g}^{+}\right) & \\
& & \mathbf{T}_{i}\left(\phi_{g}{ }^{-}\right)
\end{array}\right] .
$$

The mean-square moments of interest at present are the mean-square end-to-end distance, or dimensions, $\left\langle r^{2}\right\rangle_{0}$ obtained from skeletal bond vectors 1 , and meansquare dipole moments $\left\langle\mu^{2}\right\rangle_{0}$ obtained from bond dipole vectors $m$. The subscript zero on these quantities limits the results to chains unperturbed by long-range interactions. Such interactions, however, should have little or no effect on the mean-square dipole moments of chains in which the resultant moment in each repeating unit lies in the plane bisecting the bond angle of a skeletal atom of the chain. ${ }^{19-21}$ Since vinyl chloride chains meet this structural requirement, the subscript on $\left\langle\mu^{2}\right\rangle_{0}$ will henceforth be deleted. The advantage of this simplification will be noted in the comparison of theoretical and experimental results. For convenience, both calculated and experimental results will be expressed as the characteristic ratio $\left\langle r^{2}\right\rangle_{0} / n l^{2}$ and the dipole-moment ratio $\left\langle\mu^{2}\right\rangle / x m^{2}$. In these ratios, $n=2 x$ is the number of skeletal bonds, each of length $l$, in the chain and $x$, being the degree of polymerization, is also the number of bond dipoles, each of magnitude $m$.

The stereochemical structure of a vinyl chain may be characterized by the fraction $f_{r}$ of stereochemical replications (isotactic placements) along the chain, i.e., the fraction of units which have the same stereochemical configuration as their predecessor along the chain. For long chains, $f_{\tau}$ will be equal to the replication probability $p_{r}$, which is the probability that in the formation of the chain, the entering unit will adopt the same stereochemical configuration as the unit to which it is being added. As previously described, ${ }^{1}$ Monte Carlo methods may be employed to generate representative stereochemical sequences for any chosen value of the replication probability.

\section{CALCULATED RESULTS AND DISCUSSION}

The statistical weight factors described in the previous section have been obtained by Flory and Williams ${ }^{17}$ by qualitative estimates based on the relative sizes of the interacting atoms and groups and the magnitudes of the Coulombic interactions, and by analysis of the stereochemical equilibrium composition of 2,4-dichloro- $n$-pentane in dimethyl sulfoxide at $70^{\circ}$. Their results were corrected to $25^{\circ}$, the temperature adopted in the present study by assuming each factor to be expressible as a Boltzmann factor in the energy of the interaction with which it is associated. These adjusted values are $\eta=4.2, \tau=0.45, \omega=\omega^{\prime \prime}=$ 0.032 , and $\omega^{\prime}=0.071$. The basis for the relative magnitudes of these statistical weight factors have been discussed previously ${ }^{17}$ and can readily be seen from Fig. 2 and the corresponding discussion in the preceding section. The factor $\eta$ is considerably larger than unity because the relatively small size of the $\mathrm{Cl}$ atom makes conformations in which it is $s y n$ to $\mathrm{CH}$ of lower energy than conformations in which the larger $\mathrm{CH}_{2}$ group is syn to $\mathrm{CH}$. As would be expected, the remaining con- 


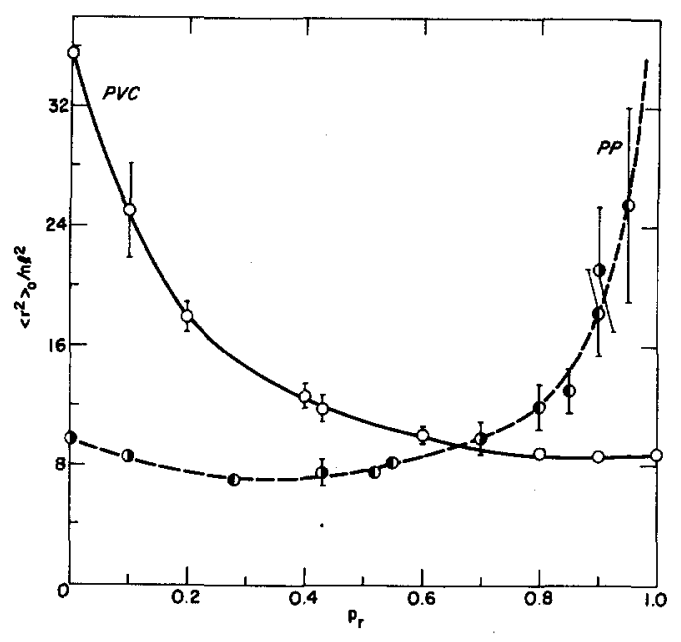

FIG. 3. The characteristic ratio $\left\langle r^{2}\right\rangle_{0} / n l^{2}$ shown as a function of the probability $p_{r}$ of replication (isotactic placement) for poly (vinyl chloride) and polypropylene of degree of polymerization $x=\frac{1}{2} n=100$ at $25^{\circ}$. In the case of stereoirregular chains, each point in this and the following figures represents the average result of calculations on a number of Monte Carlo chains having the specified value of $p_{r}$; lines through the points have been drawn with a length twice the standard deviation in that set of results. For polypropylene, left-filled circles refer to previously published results, ${ }^{1}$ and right-filled circles, to results obtained in the present study.

formation, in which both $\mathrm{Cl}$ and $\mathrm{CH}_{2}$ are $s y n$ to $\mathrm{CH}$, receives a statistical weight factor significantly less than unity. The values assigned to the factors $\omega$ and $\omega^{\prime \prime}$ are relatively small, indicating strong repulsions between $\mathrm{CH}_{2}$ groups and between $\mathrm{Cl}$ atoms, respectively. In the former instance, these repulsions are due to the relatively large size of the $\mathrm{CH}_{2}$ group, and in the latter, they are due at least in part to electrostatic repulsions between partially charged $\mathrm{Cl}$ atoms. Corresponding second-order interactions involving a $\mathrm{CH}_{2}$ group and a $\mathrm{Cl}$ atom should be less severe than those in the preceding two cases, and this is reflected in the somewhat larger value assigned to the associated statistical weight factor, $\omega^{\prime}$. Electrostatic interactions in PVC chains are thus seen to be of relatively minor importance; their only significant effect is apparently a decrease in one of the statistical weight factors, $\omega^{\prime \prime}$, characterizing second-order interactions.

On the basis of structural information on small molecules, ${ }^{22}$ the skeletal bond angles in these chains were assumed to be $112^{\circ}$, and skeletal bond vectors were specified by components $l_{x}=1.53$, and $l_{y}=l_{z}=$ 0.0 in bond coordinate systems defined as described above. The magnitude of the $\mathrm{C}-\mathrm{Cl}$ bond dipole moment $m$ was taken to be $2.00 \mathrm{D}$, the observed dipole moment of ethyl chloride in the gas phase ${ }^{23}$ and, on the basis of structural information on related compounds, ${ }^{22}$ the $\mathrm{Cl}$ atoms were assumed to be tetrahedrally bonded to the carbon atoms of the chain. In these approximations, the bond dipole vector at a carbon atom of $d$ atomic configuration has components $m_{x}=-0.668$, $m_{y}=0.943$, and $m_{z}=-1.633$; the vector at a carbon of $l$ configuration would have the sign of $m_{z}$ reversed. Sequence distributions for chains of imperfect stereochemical composition $\left(0<f_{r}<1\right)$ were obtained from sets of $x$ computer-generated random numbers which ranged from 0 to 1 in magnitude. ${ }^{24}$ The magnitude of the first number was used to specify the atomic configuration of the first unit; thereafter, occurrence of a number less than the chosen value of $p_{r}$ was taken to specify an isotactic placement (replication) and a number greater than $p_{r}$, a syndiotactic placement (inversion). Number sequences for which $f_{r}$ differed from $p_{r}$ by more than 0.05 unit were discarded except in the case of 10 or fewer numbers; in this case a maximum difference of 0.10 unit was permitted in order to obtain representative sequences. The average of the values of a configuration-dependent property calculated for five Monte Carlo chains thus generated was taken to be a representative result for vinyl chloride chains at the specified value of $p_{r}$.

Using the above information and methods, values of the characteristic ratio at $25^{\circ}$ were calculated for poly (vinyl chloride) (PVC) chains having $x=100$, over the entire range of stereochemical composition. These results are shown in Fig. 3; in this and the following figures, lines of length twice the standard deviation in the set of results have been drawn through the points in those cases in which this length exceeded the diameter of the circles used to locate the points. One set of results was obtained for $p_{r}=0.43$, which generates a slightly syndiotactic polymer, because NMR and ir spectroscopic studies by a number of workers ${ }^{25-32}$ have indicated this value for the replication probability for a "typical" sample of PVC, i.e., one prepared by free-radical polymerization in the vicinity of $50^{\circ}$. For later comparison of theory and experiment, an additional calculated result for this value of $p_{r}$ was obtained for a temperature of $155.4^{\circ}$ by adjustment of the statistical weight factors as already described. The result was $\left\langle r^{2}\right\rangle_{0} / n l^{2}=9.53$, with a standard deviation of 0.20 . In Fig. 3, rightfilled circles show, for purposes of comparison, characteristic ratios at $25^{\circ}$ calculated for polypropylene (PP) chains using the same rotational states and value of $x$, but statistical weight factors ${ }^{1}$ appropriate to this chain molecule: $\eta=1, \omega=\omega^{\prime}=\omega^{\prime \prime}=0$. (Setting the second-order factors equal to zero makes the value of $\tau$ employed immaterial.) Also represented, by leftfilled circles, are previous results ${ }^{1}$ on PP chains using the same data and methods; one Monte Carlo chain had been used to obtain each of the results shown for $p_{r}=0.28,0.52$, and 0.55 , two for $p_{r}=0.80,0.85$, and 0.95 , and five for $p_{r}=0.90$.

The very different dependence of the characteristic 
ratio on the replication probability for these two vinyl polymers is primarily due to the small size of the $\mathrm{Cl}$ atom relative to the $\mathrm{CH}_{3}$ group. This structural difference is of course quantitatively accounted for in the two sets of statistical weight factors, but it is useful to provide the following simple physical picture based on detailed examination of the calculated results. In the region of high syndiotacticity, the characteristic ratio of $\mathrm{PVC}$ is much larger than that of $\mathrm{PP}$ primarily because the small size of the $\mathrm{Cl}$ atom causes a strong preference $(\eta \gg 1)$ for extended $t t$ states about $\mathrm{CHCl}-$ $\mathrm{CH}_{2}-\mathrm{CHCl}$ bond pairs in syndiotactic sequences in PVC chains. ${ }^{17}$ In the case of high isotacticity, the characteristic ratio is much higher for PP because the relatively large size of the pendant $\mathrm{CH}_{3}$ groups effectively prevents significant departures from extended helical conformations in isotactic sequences $(\omega=0){ }^{1}$ (For PP chains, reasonable alteration of the locations of rotational states in instances of severe steric re-

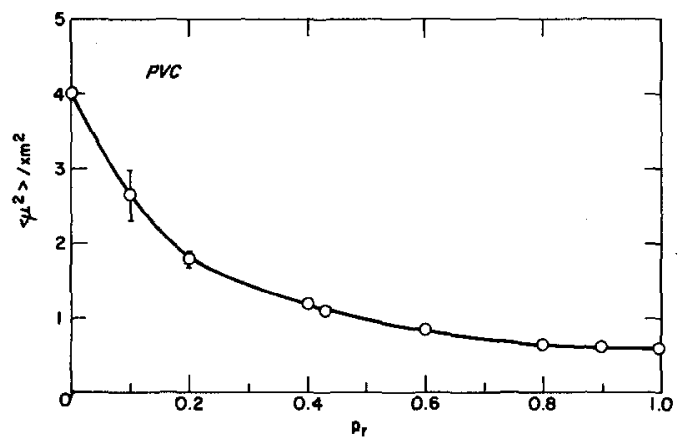

FIG. 4. The dependence of the dipole-moment ratio $\left\langle\mu^{2}\right\rangle / x m^{2}$ on the replication probability for poly(vinyl chloride) chains of $x=100$ at $25^{\circ}$.

pulsions and corresponding adjustment of the statistical weight factors results primarily in a large decrease in the value of the characteristic ratio predicted for highly isotactic chains., ${ }^{1,2,18}$ This would not, however, qualitatively change the marked differences between the calculated results for PVC and PP chains cited above.)

Figure 4 shows the dependence of the dipole-moment ratio on $p_{r}$ for PVC chains having $x=100$ at $25^{\circ}$. The predicted large decrease in the value of this ratio with increasing isotacticity is due to the fact that lowenergy conformations of isotactic sequences in these chains give rise to greater mutual attenuation than do low-energy conformations $(t t)$ of syndiotactic sequences.

Figures 5 and 6 show the dependence of the characteristic ratio on the degree of polymerization $x$ for vinyl chloride and propylene chains, respectively, at $25^{\circ}$; Fig. 7 shows the dependence of the dipole-moment ratio on $x$ for vinyl chloride chains, at the same temper-

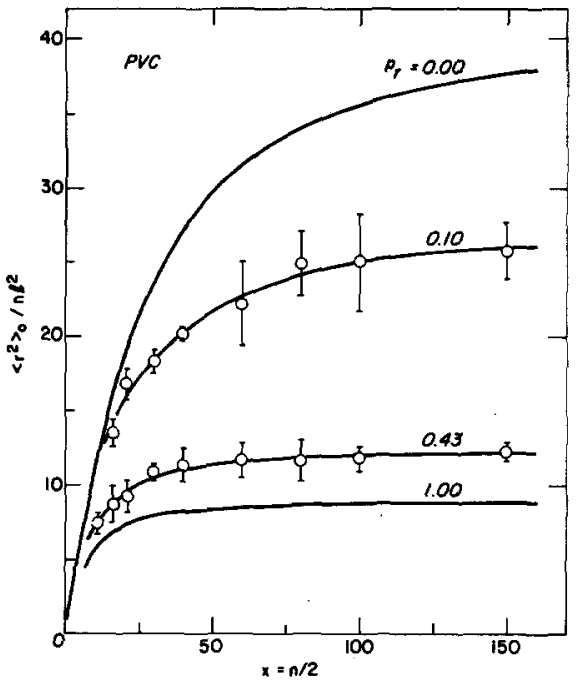

FIG. 5. The characteristic ratio of poly (vinyl chloride) plotted against the degree of polymerization. In this and the following figures the temperature is $25^{\circ}$ and each curve is labeled with the value of the replication probability $p_{r}$ used to generate the stereochemical structure of the chains of varying degrees of polymerization.

ature. In these figures, each curve is labeled with the value of $p_{r}$ used to generate the set of chains of varying degree of polymerization. For purposes of clarity, not all points used to locate these curves have been shown. As expected, the larger the value of the characteristic ratio or dipole-moment ratio, the more protracted the rate at which the ratio converges to its limiting value for large $x$. It should be noted that since a typical vinyl chloride polymer has a value of $p_{r}$ of approximately $0.43,,^{25-32}$ its characteristic ratio and dipole-

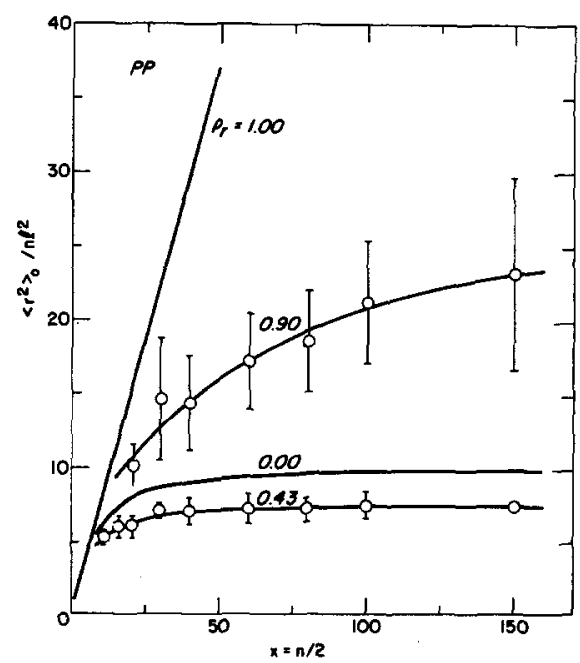

FIG. 6. The characteristic ratio of polypropylene plotted against the degree of polymerization for selected values of $p_{r}$. 


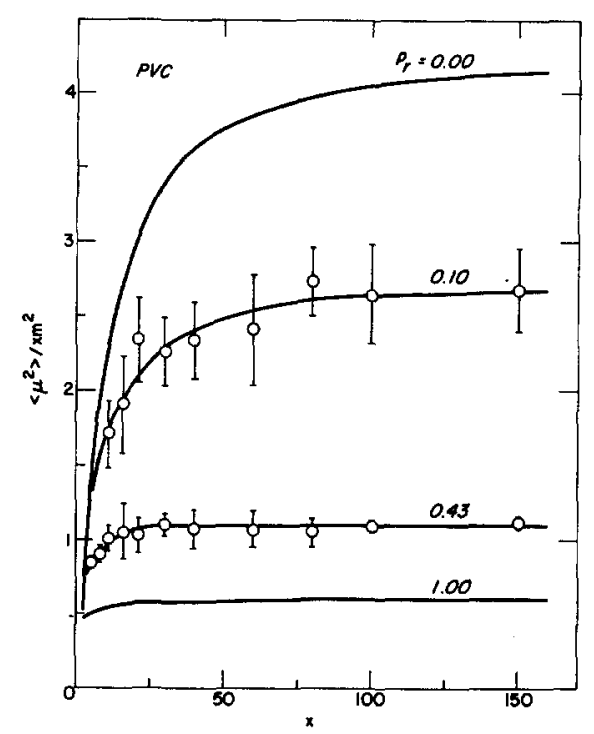

Fyg. 7. The dipole-moment ratio of poly (vinyl chloride) plotted against the degree of polymerization for selected values of $p_{r}$.

moment ratio should be essentially independent of $x$ for values of $x$ exceeding approximately 100 .

The dependence of $\left\langle\boldsymbol{r}^{2}\right\rangle_{0}$ and $\left\langle\mu^{2}\right\rangle$ on the statistical weight factors was calculated for PVC chains having $x=100$, at values of the factors appropriate to $25^{\circ}$, for values of $p_{r}$ of $0.00,0.10,0.43,0.90$, and 1.00 . For purposes of economy, the calculations for $p_{r}=$ $0.10,0.43$, and 0.90 were carried out on the single Monte Carlo chain judged on the basis of the previous calculations to be most representative of chains of each of these stereochemical compositions. The results are given in Columns 3-7 of Table I. Temperature coefficients of $\left\langle r^{2}\right\rangle_{0}$ and $\left\langle\mu^{2}\right\rangle$, calculated from these results in the usual manner, ${ }^{33}$ assuming the statistical weight factors to be Boltzmann factors, are given in the final column of the table.

Temperature coefficients of $\left\langle r^{2}\right\rangle_{0}$ have also been calculated $^{1,2}$ for PP chains in which the requirement $\omega=\omega^{\prime}=\omega^{\prime \prime}=0$ was relaxed on the basis of the displacement of the locations of several rotational states, as already mentioned. For values of $p_{r}$ increasing from 0.0 to $1.0,-10^{3} d \ln \left\langle r^{2}\right\rangle_{0} / d T$ was predicted to increase monotonically from approximately 0.7 to 6.5 , the reverse of the dependence on $p_{r}$ calculated for PVC chains. Thus there is a strong decrease in $\left\langle r^{2}\right\rangle_{0}$ with increasing temperature at low values of $p_{r}$ in the case of PVC and at high values of $p_{r}$ in the case of PP. This dependence is primarily due to the fact that in syndiotactic sequences in PVC chains and in isotactic sequences in PP chains, the low-energy conformations are very extended and therefore departures from these conformations brought about by an increase in temperature decrease the dimensions markedly. Since there is also less mutual attenuation of bond dipoles in the low- energy conformations of syndiotactic PVC chains, $\left\langle\mu^{2}\right\rangle$ also decreases significantly with increase in temperature at low values of $p_{r}$.

\section{COMPARISON OF THEORY AND EXPERIMENT}

The experimental studies which may be used to evaluate the above calculated results are somewhat limited and, unfortunately, have almost invariably been carried out on polymers of uncharacterized stereochemical composition. These polymers have usually been prepared by free-radical polymerization in the vicinity of $50^{\circ}$ and therefore, unless otherwise specified, the experimental results will be compared with calculated results which pertain to $p_{r} \cong 0.43$. In the following preliminary comparison of theoretical and experimental results, it will be assumed that changes in conformational energy due to changes in dielectric constant of the medium are negligibly small. This assumption, based on the above analysis of statistical weight factors, is implicit in the use of statistical weight factors determined in one solvent for the prediction of statistical properties in a variety of other solvents. Since the polymers studied were reported to be of relatively high molecular weight, the values of $\left\langle r^{2}\right\rangle_{0} / n l^{2}$ and $\left\langle\mu^{2}\right\rangle / x m^{2}$ in the limit of large $x$ will be required; as mentioned previously, results calculated for $x=100$ are suitable for this purpose.

The most reliable determination of the characteristic ratio of these chains is based on a study ${ }^{34}$ of a number of fractions of PVC obtained from a polymer prepared by free-radical initiation at $50^{\circ}$. Phase equilibrium measurements on these fractions in benzyl alcohol indicated that the $\theta$ point for this system is $155.4^{\circ}$; under these conditions the dimensions of these molecules are unperturbed by long-range interactions, i.e., excluded volume effects. ${ }^{35}$ Molecular weights and intrinsic viscosities determined for these fractions at the $\theta$ point, along with the value $e^{36,37} 2.5 \times 10^{21}$ for the hydrodynamic constant $\Phi,{ }^{35}$ indicate a value of $\left\langle r^{2}\right\rangle_{0} / n l^{2}$ of 9.75 at $155.4^{\circ}$. This experimental value of the characteristic ratio is in excellent agreement with that calculated for $p_{r}=0.43$ at the same temperature: 9.53, with a standard deviation of 0.20 . Similar studies ${ }^{38}$ have been carried out on PVC fractions obtained from a series of polymers which had been prepared at four temperatures decreasing from 50 to $-15^{\circ}$ and which were reported ${ }^{29}$ to have replication probabilities decreasing from 0.46 to 0.36 . An approximate method ${ }^{39,40}$ was used to determine the composition of tetrahydrofuran-water mixtures which would approximate the $\theta$ point at $30^{\circ}$ for each fraction studied. Molecular weights and intrinsic viscosities measured under these conditions gave characteristic ratios at $30^{\circ}$ which increased from 12.0 to 16.3 as $p_{r}$ decreased from 0.46 to 0.36 . These results are in reasonable agreement with the corresponding increase, 11.4 to 13.3 , predicted by theory. 
TABLE I. The effect of the statistical weight factors and temperature on the mean-square unperturbed dimensions and dipole moments of poly (vinyl chloride). a $^{2}$

\begin{tabular}{cccccccc}
\hline$\left\langle V^{2}\right\rangle$ & $p_{r}$ & $\partial \ln \left\langle V^{2}\right\rangle / \partial \ln \eta$ & $\partial \ln \left\langle V^{2}\right\rangle / \partial \ln \tau$ & $\partial \ln \left\langle V^{2}\right\rangle / \partial \ln \omega$ & $\partial \ln \left\langle V^{2}\right\rangle / \partial \ln \omega^{\prime}$ & $\partial \ln \left\langle V^{2}\right\rangle / \partial \ln \omega^{\prime \prime}$ & $10^{3} d \ln \left\langle V^{2}\right\rangle / d T$ \\
\hline$\left\langle r^{2}\right\rangle_{0}$ & 0.00 & 1.218 & -0.051 & -0.012 & -0.107 & -0.040 & -7.55 \\
& 0.10 & 0.850 & -0.068 & -0.008 & -0.088 & -0.005 & -5.20 \\
& 0.43 & 0.275 & -0.019 & -0.036 & -0.015 & 0.032 & -1.55 \\
& 0.90 & 0.009 & -0.074 & -0.073 & -0.077 & -0.042 & -2.25 \\
& 1.00 & -0.029 & -0.097 & -0.071 & -0.101 & -0.066 & -2.60 \\
$\left\langle\mu^{2}\right\rangle$ & 0.00 & 1.552 & -0.033 & -0.007 & -0.214 & -0.030 & -9.88 \\
& 0.10 & 1.116 & -0.030 & -0.004 & -0.101 & 0.008 & -6.30 \\
& 0.43 & 0.443 & -0.005 & -0.014 & -0.043 & 0.085 & -1.71 \\
& 0.90 & 0.131 & -0.042 & -0.026 & -0.040 & 0.110 & -0.63 \\
& 1.00 & 0.106 & -0.061 & -0.033 & -0.068 & 0.100 & -0.50 \\
\hline \hline
\end{tabular}

* Calculated for a degree of polymerization of 100 , temperature of $25^{\circ}$, and values of the statistical weight factors given in the text.

There have also been a large number of studies ${ }^{41-45}$ of the viscosity of PVC chains in solvents in which long-range interactions are present. Although the extrapolation procedures ${ }^{46}$ used to estimate values of the characteristic ratio from such data have been severely criticized as being unreliable, ${ }^{47}$ we include a summary of such results for the purpose of completeness. The values of the characteristic ratio thus obtained in a wide variety of solvents and in the vicinity of $25^{\circ}$, yield the average result $8.2 \pm 1.1$ for $\Phi=2.5 \times$ $10^{21}$. This average value is in only fair agreement with theory and with the previously quoted, more reliable experimental results. It is perhaps worth noting, however, that one ${ }^{44}$ of these studies reports a value of $d \ln \left\langle r^{2}\right\rangle_{0} / d T$ from such viscosity measurements at a number of temperatures. Their result, $-1.5 \times 10^{-3}$ $\operatorname{deg}^{-1}$, is in almost exact agreement with the result shown in Table I for $p_{r}=\mathbf{0 . 4 3}$.

There have been a number of studies ${ }^{48-50}$ of the dipole moments of PVC chains which, because of the previously mentioned absence of any effect of longrange interactions on $\left\langle\mu^{2}\right\rangle$, should provide results pertinent to the comparison of theory and experiment. Unfortunately, the considerable disagreement among the available experimental results makes reliable comparison of theoretical and experimental values of the dipole-moment ratio impossible at the present time. The results to date, however, seem to indicate values of $\left\langle\mu^{2}\right\rangle / \mathrm{xm}^{2}$ of approximately $0.4-0.8$ in the vicinity of $25^{\circ}$; this would be significantly lower than the value calculated for $p_{r}=0.43$ and $25^{\circ}: 1.10$, with a standard deviation of 0.03 . It is important to mention that in the most detailed of these studies, ${ }^{49}$ dipole moments were measured for a series of PVC fractions which, on the basis of their solubility characteristics, would be expected to have increasing crystallizability; since the polymer had been prepared by free-radical initiation, this would suggest increasing syndiotacticity.
These samples clearly showed an increase in dipole moment with presumed increase in syndiotacticity, in agreement with theory.

A more meaningful comparison of theoretical and experimental values of the unperturbed dimensions and dipole moments would obviously require additional careful measurements on vinyl chloride polymers of well-characterized stereochemical composition.

\section{ACKNOWLEDGMENT}

It is a pleasure to acknowledge that this research was supported by the National Science Foundation through Grant GP-16028.

1 P. J. Flory, J. E. Mark, and A. Abe, J. Am. Chem. Soc. 88, 639 (1966).

2 A. Abe, Polymer J. 1, 232 (1970).

${ }^{3}$ Y. Fujiwara and P. J. Flory, Macromolecules 3, 280 (1970).

${ }^{4}$ K. Nagai, J. Chem. Phys. 47, 2052 (1967).

${ }^{5}$ P. J. Flory, R. L. Jernigan, and A. E. Tonelli, J. Chem. Phys. 48, 3822 (1968).

${ }^{6}$ Y. Abe, A. E. Tonelli, and P. J. Flory, Macromolecules 3, 294 (1970).

7 A. Abe, J. Am. Chem. Soc. 90, 2205 (1968).

${ }^{8}$ A. Abe, J. Am. Chem. Soc. 92, 1136 (1970).

${ }^{9}$ Y. Fujiwara and P. J. Flory, Macromolecules 3, 288 (1970).

${ }^{10}$ A. E. Tonelli; Y. Abe, and P. J. Flory, Macromolecules 3, 303 (1970).

11 P. J. Flory and J. D. Baldeschwieler, J. Am. Chem. Soc. 88, 2873 (1966).

${ }_{12} \mathrm{P}$. J. Flory and Y. Fujiwara, Macromolecules 2, 315, 327 (1969)

${ }^{13}$ Y. Fujiwara and P. J. Flory, Macromolecules 3, 43 (1970). ${ }^{14}$ Y. Abe and P. J. Flory, J. Chem. Phys. 52, 2814 (1970).

${ }_{15}$ A. D. Williams, J. I. Brauman, N. J. Nelson, and P. J. Flory, J. Am. Chem. Soc. 89, 4807 (1967).

${ }_{16}$ A. D. Williams and P. J. Flory, J. Am. Chem. Soc. 91, 3111 (1969).

${ }^{17}$ P. J. Flory and A. D. Williams, J. Am. Chem. Soc. 91, 3118 (1969).

${ }_{18}$ P. J. Flory, Statistical Mechanics of Chain Molecules (Interscience, New York, 1969).

${ }^{19} \mathrm{~J}$. Marchal and H. Benoit, J. Chim. Phys. 52, 818 (1955); J. Polymer Sci. 23, 223 (1957).

${ }_{20}$ W. H. Stockmayer, Pure Appl. Chem. 15, 539 (1967). 
${ }^{21} \mathrm{~K}$. Nagai and T. Ishikawa, Polymer J. 2, 321 (1971).

${ }^{22}$ H. J. M. Bowen (Ed)., Chem. Soc. (London), Spec. Publ. 11 (1958); L. E. Sutton (Ed.), Chem. Soc. (London), Spec. Publ. 18 (1965).

${ }_{23}$ A. L. McClellan, Tables of Experimental Dipole Moments

(W. H. Freeman, San Francisco, Calif., 1963).

${ }_{24}$ IBM subroutine RANDU (SSP $/ 360$, version 2).

${ }_{25}$ S. Satoh, J. Polymer Sci. Pt. A, 2, 5221 (1964).

${ }^{26}$ W. C. Tincher, Makromol. Chem. 85, 20 (1965).

${ }^{27} \mathrm{~J}$. Bargon, K. H. Hellwege, and U. Johnson, Makromol. Chem. 95, 187 (1966)

${ }^{28}$ S. Enemoto, M. Asahina, and S. Satoh, J. Polymer Sci. Pt. A1 4, 1373 (1966).

${ }^{29}$ A. Nakajima, F. Hamada, and S. Hayashi, Makromol. Chem. 95, 40 (1966).

${ }^{30}$ F. A. Bovey, F. P. Hood, E. W. Anderson, and R. L. Kornegay, J. Phys. Chem. 71, 312 (1967).

${ }^{31}$ L. Cavalli, G. C. Borsini, G. Carraro, and G. Confalonieri, J. Polymer Sci. Pt. A1 8, 801 (1970).

${ }_{32}$ F. Heatley and F. A. Bovey, Macromolecules 2, 241 (1969).

${ }^{33}$ P. J. Flory, V. Crescenzi, and J. E. Mark, J. Am. Chem. Soc. 86, 146 (1964).

${ }^{34}$ M. Sato, Y. Koshiishi, and M. Asahina, J. Polymer Sci. Pt. B 1, 233 (1963).

${ }_{35}$ P. J. Flory, Principles of Polymer Chemistry (Cornell U. P., Ithaca, N.Y., 1953).
${ }^{36}$ D. McIntyre, A. Wims, L. C. Williams, and L. Mandelkern, J. Phys. Chem. 66, 1932 (1962)

${ }^{37}$ G. C. Berry, J. Chem. Phys. 46, 1338 (1967).

${ }^{38}$ A. Nakajima and K. Kato, Makromol. Chem. 95, 52 (1966).

${ }^{39}$ H.-G. Elias, Makromol. Chem. 33, 140 (1959).

${ }^{40}$ H.-G. Elias, M. Dobler, and H.-R. Wyss, J. Polymer Sci. 46, $264(1960)$.

${ }^{41}$ See the compilation of such results by $M$. Kurata, M. Iwama and K. Kamada, in Polymer Handbook, edited by J. Brandup and E. H. Immergut (Interscience, New York, 1966).

${ }^{42}$ A. Nakazawa, T. Matsuo, and H. Inagaki, Bull. Inst. Chem. Res. Kyoto Univ. 44, 354 (1966).

${ }^{43}$ M. Kolínský, M. Ryska, M. Bohdanecký, P. Kratochvíl, K. Šolc, and D. Lím, J. Polymer Sci. Pt. C 16, 485 (1967).

${ }^{44}$ M. Bohdanecký, V. Petrus, and P. Kratochvil, Collection Czech. Chem. Commun. 34, 1168 (1968).

${ }^{45}$ L. A. Utracki, Macromolecular Preprints, XXIII International Congress of Pure and Applied Chemistry, 1037, 1971.

${ }^{46}$ See, for example, M. Kurata and W. H. Stockmayer, Fortschr. Hochpolymer Forsch. 3, 196 (1963).

${ }_{47}$ P. J. Flory, Makromol. Chem. 98, 128 (1966)

${ }^{48}$ Y. Imamura, J. Chem. Soc. Japan 76, 217 (1955)

${ }^{49}$ R. J. W. Le Fèvre and K. M. S. Sundaram, J. Chem. Soc. 1962,1494

${ }^{50}$ A. Kotera, M. Shima, N. Fujisaki, and T. Kobayashi, Bull. Chem. Soc. Japan 35, 1117 (1962).

\title{
Random-Coil Dimensions and Dipole Moments of $p$-Chlorostyrene Chains
}

\author{
J. E. MARK \\ Department of Chemistry and the Macromolecular Research Center, University of Michigan, Ann Arbor, Michigan 48104
}

(Received 23 July 1971)

\begin{abstract}
Mean-square dimensions and dipole moments of $p$-chlorostyrene chains $\mathrm{CH}_{3}-\left[\mathrm{CH}\left(p-\mathrm{C}_{6} \mathrm{H}_{4} \mathrm{Cl}\right)-\mathrm{CH}_{2}-\right]_{x} \mathrm{H}$ have been calculated as a function of their stereochemical structure, degree of polymerization $x$, and temperature. Theoretical arguments and experimental evidence indicate that p-chlorostyrene and styrene chains differ little in conformational energy. Therefore, the present investigation employs conformational energies of styrene chains recently obtained by Flory and co-workers from analysis of the stereochemical equilibrium compositions of styrene dimers and trimers. The calculations indicate that at large $x$ the dimensions of $p$-chlorostyrene, or styrene, chains and the dipole moments of $p$-chlorostyrene chains should increase with increasing isotacticity and decreasing temperature. The marked differences in the configurational characteristics of $p$-chlorostyrene and vinyl chloride chains are shown to result from the large difference in size between $p-\mathrm{C}_{6} \mathrm{H}_{4} \mathrm{Cl}$ groups and $\mathrm{Cl}$ atoms. Theoretical results calculated for large $x$ are, in general, in satisfactory agreement with published experimental results on poly ( $p$-chlorostyrene). Several experimental results, as yet unconfirmed, seem however to evade interpretation in terms of the present model. Additional experimental investigations would be required to resolve this discrepancy and to provide a more definitive test of the calculated results.
\end{abstract}

\section{INTRODUCTION}

Calculations of the random-coil dimensions and dipole moments of vinyl chloride chains $\mathrm{CH}_{3}-\left[\mathrm{CHCl}-\mathrm{CH}_{2}-\right]_{x} \mathrm{H}$ have been reported in the preceding paper. ${ }^{1}$ These calculations constitute the only interpretation of the dipole moments of a vinyl chain molecule using a realistic model, a rotational isomeric state representation with neighbor interactions. Vinyl chloride oligomers and polymers are, however, unusual vinyl chains in that the small size of the pendant substituent $\mathrm{R}, \mathrm{a} \mathrm{Cl}$ atom, gives these molecules access to a larger number of conformations 1,2 $^{12}$ than are available to "typical" vinyl chains, i.e., those having substituents the size of a methyl group or larger ${ }^{3-7}$ For example, an isotactic polymer of vinyl chloride would not be expected ${ }^{1,2}$ to exhibit as strong a preference for $3_{1}$ helical sequences as would isotactic polymers of propylene or styrene. ${ }^{3-7}$

It is therefore of considerable interest to extend the earlier calculations to vinyl oligomers and polymers having significant dipole moments and sufficiently large $R$ substituents to limit, by steric interactions, the conformations accessible to the chain backbone. Chains of para $(p)$-chlorostyrene units $\mathrm{CH}_{3}-\left[\mathrm{CH}\left(p-\mathrm{C}_{6} \mathrm{H}_{4} \mathrm{Cl}\right)\right.$ $\left.\mathrm{CH}_{2}-\right]_{x} \mathrm{H}$ are ideally suited for this purpose. Because of the nature of the bonding ${ }^{8}$ and the symmetry of the para isomer, the resultant group dipole in each repeat unit should lie, to a very good approximation, along the $\mathrm{C}^{\alpha}-\mathrm{C}^{\beta}$ bond, as shown in Fig. 1. Furthermore, in both vinyl chloride and $p$-chlorostyrene chains, this dipole 